\title{
Novel continuously variable transmission mechanism
}

\author{
Abbas Olyaei ${ }^{1}$ \\ (c) Springer Nature Switzerland AG 2019
}

\begin{abstract}
Continues changing of gear ratio is accomplished by continuously variable transmission (CVT) gearboxes that perfectly matches the torque and rotational speed of engine with output conditions. Developing CVTs for better performance and make it more reliable is ongoing effort. Here we present new ratcheting CVT mechanism that is similar to cam based CVT but instead cam it has a free shaft. New CVT is totally mechanical, frictionless and self-adjustable. Its automatic feature makes it cost effective and reliable. Wide gear ratio is achievable easily and simple structure make it more feasible. In opposite to conventional belt-type or toroidal CVTs, this new CVT doesn't use friction force, so its energy transfer efficiency improved and made it durable.
\end{abstract}

Keywords CVT · Continuously variable transmission · Gearbox · Power transmission · Gear ratio · Frictionless

\section{Introduction}

Since the earlier years of automobile invention, it has been known that power transmission unite has crucial role on total performance of machine [1-5]. Gearbox is the heart of power transmission unite that matches the required torque and rotational speed of the engine with output conditions. Common step gearboxes just present some predesigned transmission ratios cold not accomplish desired performance. Purpose of a stepless transmission is achieved by CVT gearboxes which are able to change the transmission ratio continuously from minimum to maximum. Applying CVT let car to goes in proper speed-torque and engine to operates in fixed point. This functionality lead to optimum performance of engine and fuel economy [6-9] as make driving easier and more comfortable [10]. Moreover, applications of CVTs to wind power systems and mechanical press machine have been proposed [11, 12].

There are many kinds of CVTs: Spherical CVT, Hydrostatic CVT, E-CVT, Toroidal CVT, Power-split CVT, Belt CVT, Chain CVT, Ball-type toroidal CVT, Milner CVT, etc. The main mechanical variants include: friction, ratcheting, hydrostatic, and positive drive $[13,14]$. In the conventional friction-based belt-type CVTs or sphere and rollers type CVTs [14], high forces between the pulleys and belt or between the sphere and rollers is necessary for operation, so there is significant power loss because of continues bending of belt [15-17].

Maximum transmissible torque is limited by slides of belt or rollers $[18,19]$. Even toroidal CVTs that are the most promising CVT with high torque capacity of transmissions is just obtained by coupling together in a series scheme $[11,20]$. Other novel CVT designs could be found in publications, are basically friction based [21].

As nearly all types of CVTs need a separate control unites, that changes the gear ratio properly, transient dynamics of CVT is one of the major concerns in design and modelling of the control unite [22-24].

Many different designs for ratcheting CVTs have been proposed. Between them Cam based CVT is the most cited one [25-27].

As seen in Fig. 1 the cam based CVT takes a rotational input from cam (part1), converts it to an oscillating motion of varying amplitude in followers(part 2), and then rectifies this motion through a number of one-way clutches (part 3 and 4) to a rotational output(part5). Cam based

\footnotetext{
Abbas Olyaei, aolyaei@alumni.ut.ac.ir|'Department of Mechanics, University of Tehran, Tehran, Iran.
} 


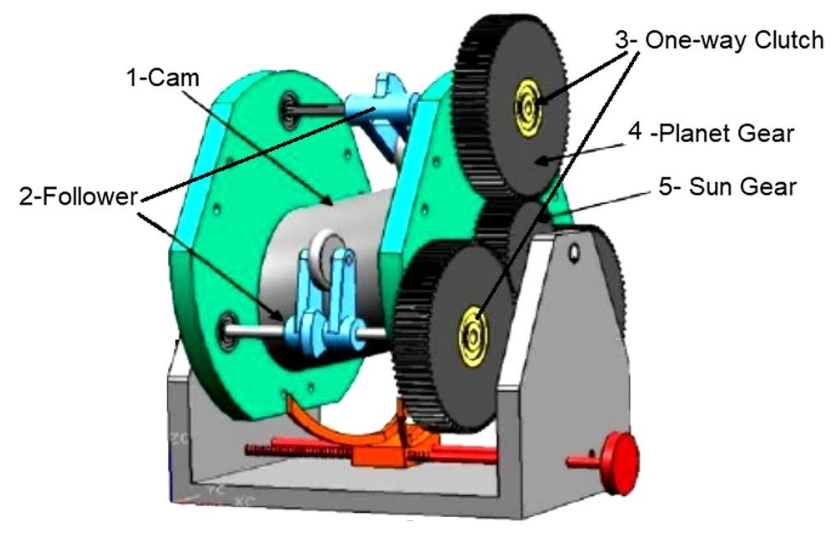

Fig. 1 Cam based CVT main concept [19]

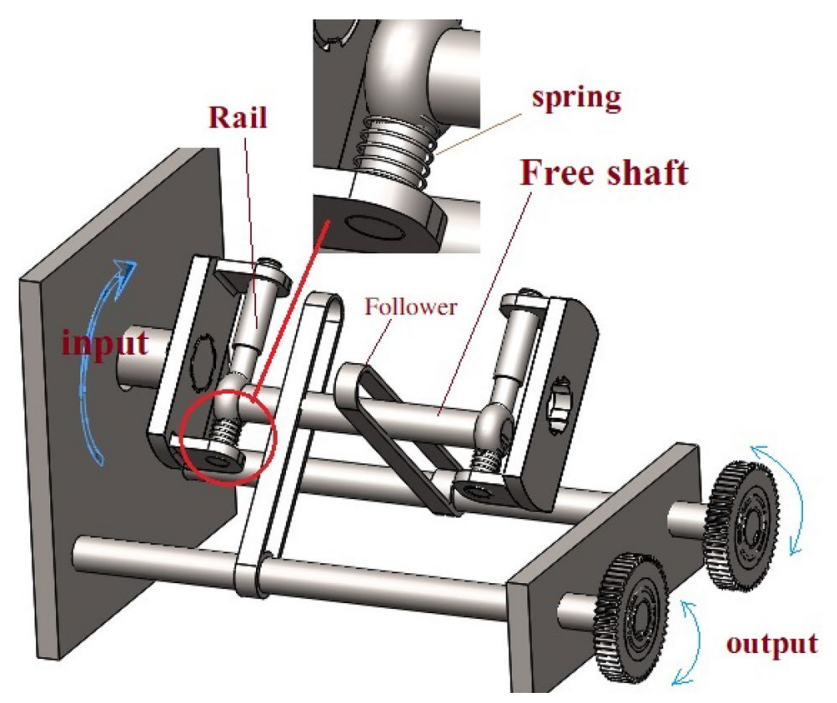

Fig. 2 New CVT concept

CVT received many developments for example the one that proposed in [26] overcome previous problems in belt-type CVTs such as a limit of maximum transmission torque. It also has less power loss and able to change the transmission ratio in wider range and act as an IVT (infinite variable transition). By the way this cam based CVT steel need external controller to change transmission ratio and may be limited by the high-speed input that repulse the followers and failure the power transition.

Here we propose new CVT mechanism based on patent "US 9,816,593 B2" that has been patented in 2017.

As seen in Fig. 2 new CVT is so similar to cam based CVT but instead of cam it has a free shaft that transfer rotational input to oscillatory motion in followers. Free shaft is free to move on rail in radial direction. This feature allows CVT to change transmission ratio automatically. There are two main difference between new idea and cam based
CVT. First the new CVT has no cam, instead of cam it takes the benefit of free shaft as a power transfer unit. Second the cam based CVT need some springs to keep the followers in tight contact with cam, but in new CVT the followers are connected to free shaft in proper manner that does not need any spring. Also as described in next sections new CVT has different functionality.

\section{Novel CVT or free-shaft CVT}

Figure 2 shows the concept of new CVT mechanism with two followers. New CVT takes a rotational input from rails. Rails rotate the free-shaft around the main axis of sketch. Then rotational motion of free-shaft is converted to oscillatory motion of varying amplitude in followers. Finally, this oscillatory motion is rectified through a number of one-way clutches to a rotational output.

A complete design of new CVT with four followers is shown in Fig. 3. As described above the oscillation of followers are collected in planetary gear set by help of oneway clutches. At the output we set a spring clutch to make the output smoother.

In the case of driving in specific ratio (constant speed), the radial position of free-shaft on rail will remain unchanged. Radial position of free-shaft determines transmission ratio. In other words, one specific transmission ratio exactly related to specific radial position of freeshaft on rail.

Changing the transmission ratio is done by changing the radial position of free- shaft on rail. This change is automatically done by the help of centrifugal force that wants to push the free-shaft out.

There is a spring on rail to push free-shaft toward the center of sketch, as free-shaft get rotate quicker it will push the spring a way and move toward the perimeter.

\section{Detailed description of CVT functionality}

The operation of the instant transmission is based on the oscillation of follower that can oscillate with constant angular velocity but with different amplitudes. Different amplitudes are correspondence to different linear velocity of follower's arm. This feature allows CVT to produce various transmission ratios at output.

Rotational frequency of the free-shaft is equal to the rotational frequency of input. The free-shaft is free to radially move on rails from the center to perimeter. Its position is determining the transmission ratio. During rotation, free shaft experience centrifugal force and try to go toward the perimeter by pressing springs which mounted on rails. 
Fig. 3 Four followers CVT

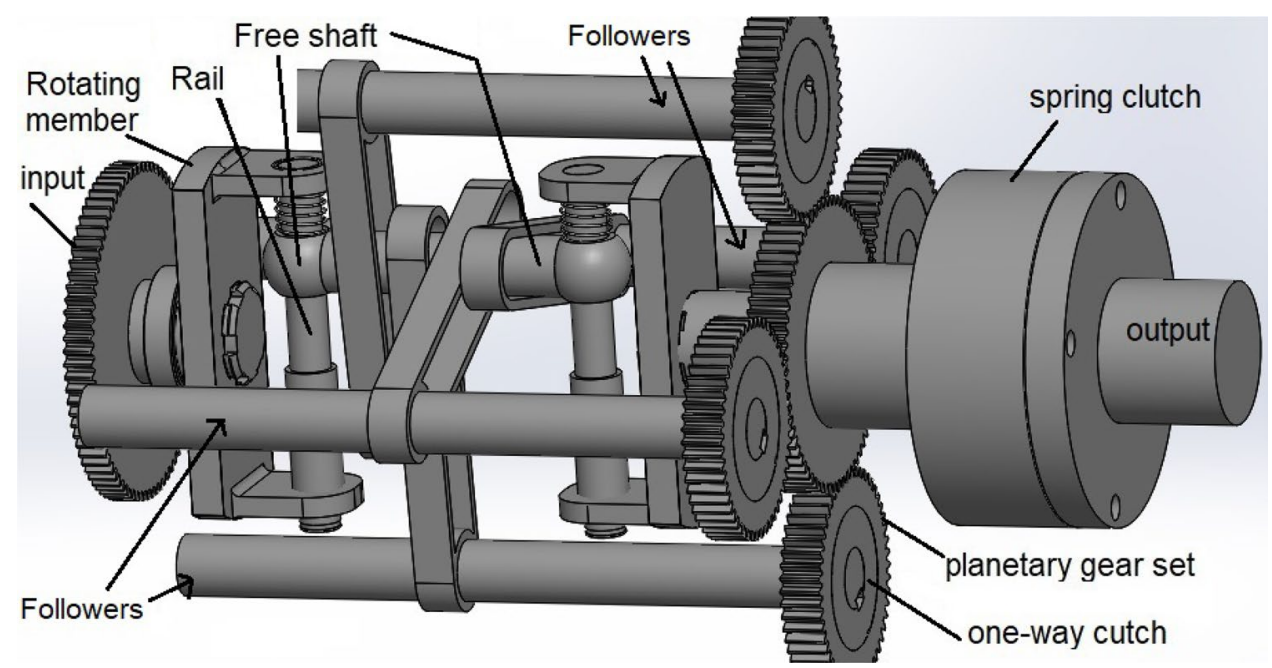

Followers are set around the free shaft. Each follower's arm has channel that allows free shaft to displace along it. Now by rotating free shaft, the followers do oscillation. Each follower connected to planetary gear by means of one-way clutch. Finally, rotation be collected in sun gear and sun gear is connected to output shaft through the spring clutch.

This CVT at least needs two followers to work properly. This is because of the structure of spring clutch. Because in every cycle of rotation, each follower is just engaged during the half time of cycle so in the case of just using one follower, the spring clutch can be turn backward during the time that the follower is not engaged. Proper CVT can work with four followers. As the number of followers increase, the output gets uniform.

Changing the ratio of transmission is automatically done in this CVT. It means that the CVT mechanism could change it self interactively.

There is a straight relation between output rotation speed and oscillation amplitudes of followers. As the free shaft go farther radius the oscillation amplitude of followers gets bigger so its planetary gear rotates faster and produce higher transmission ratio. If output is limited to rotate in specific velocity, it forces the followers to reduce its oscillation amplitude, in turn the follower forces the free shaft to get back to the smaller radius by the help of springs. So, the transmission ratio be adjusted automatically based on the road conditions and there is no need for any other control system. This means our CVT is totally self-adjustable.

The spring clutch is a key component to CVT work properly. First it covers the gap between the followers that are in different operational phases. Second it compensates the output velocity fluctuations that is occurred because of the oscillation nature of the CVT mechanism and produce uniform output. Third in sudden transmission ratio changes like as sudden breaks, the spring clutch gives CVT enough freedom to rotate a little and get adjusted. In order to help CVT work better one can add another spring clutch in input shaft.

\section{Calculation of transmission ratio}

We have to notice that each follower is engaged in limited angle that is shown in Fig. 4. So, we present the calculation of transmission ratio in two sections: in first section we just drive the maximum ratio of transmission for the case of zero rail angle $\alpha=0$ then in second section we will drive full description of transmission ratio.

\subsection{Maximum transmission ratio}

This CVT can works in both directions. For both directions of input rotation, there is just one direction output. It has two different transmission ratios for two different input directions. As shown in Fig. 5 the transmission could occur in point $A$ or in point $B$. These points refer to the positions of free shaft on rail. For one designed CVT, as one-way clutches are being set in determined rotational direction, if the input is rotating in clockwise then the free-shaft will push the follower's arm in point $A$ and if input is rotating in counter clockwise then the free-shaft will push follower's arm in point $B$. We call them, mode $A$ and mode $B$.

Line $C$ shows the center of rail that is the center of input rotation. For different input directions, the follower is just engaged in one of the positions $A$ or $B$.

Mode $A$ : In this mode, free-shaft will engage the follower, at point $A$. If $\omega_{i}$ is input angular velocity, $\omega_{0}$ is follower's shaft angular velocity and is considered as output. Free-shaft placed in distance $\Delta$ from the center of input rotation (line $\mathrm{C}$ ) and the distance between the 
Fig. 4 Transversal cross section view that indicate follower engaging angle in mode $B$
Fig. 5 Two operational modes of CVT: left side shows Mode B and right side shows Mode $A$
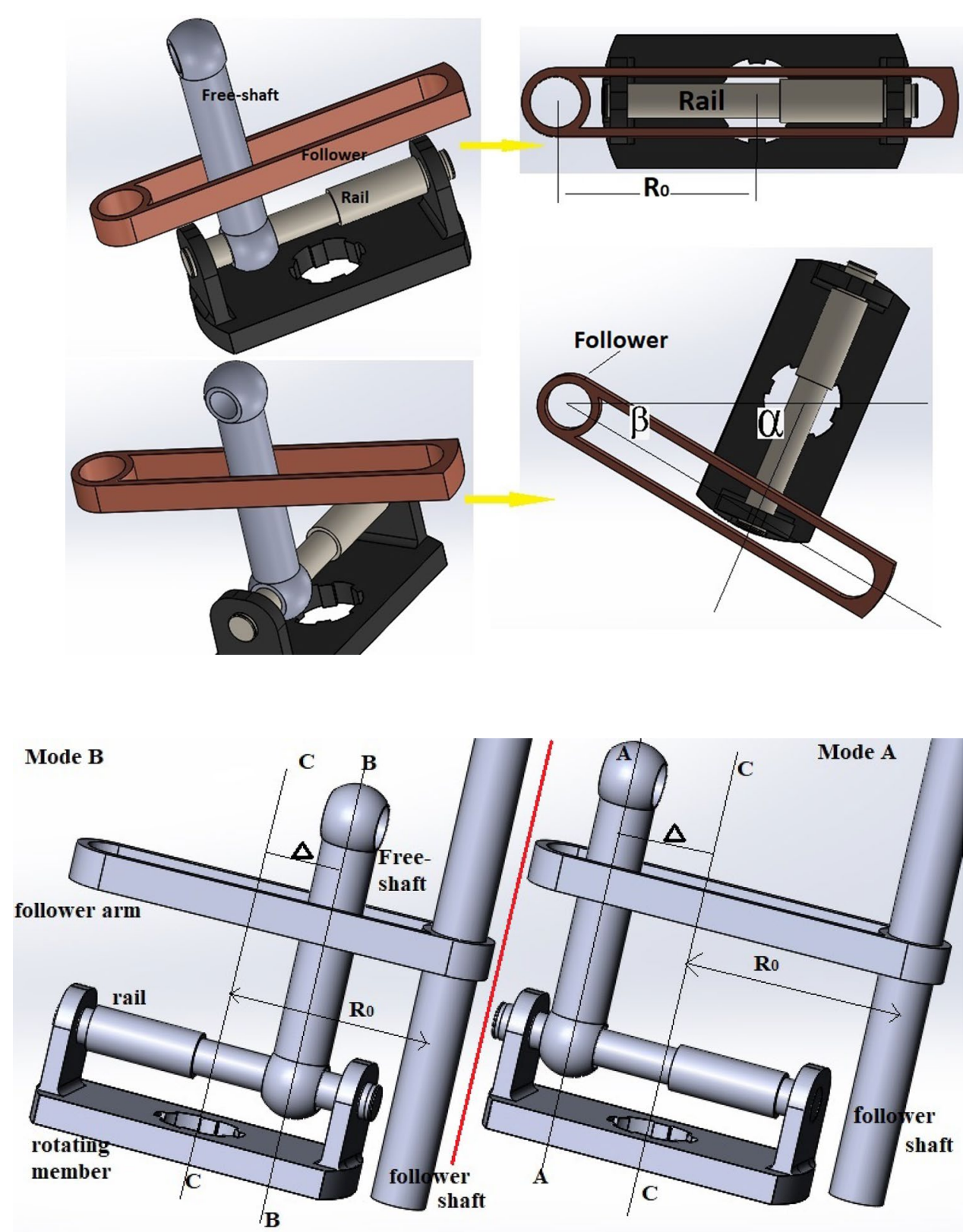

center of rotating member and the center axis of follower's shaft is $R_{0}$. Transmission ratio is:

$\frac{\omega_{\mathrm{o}}}{\omega_{\mathrm{i}}}=\frac{\Delta}{\mathrm{R}_{\mathrm{o}}+\Delta}$

Mode B: here the free-shaft will engage the follower, at point $B$. For $B$ mode the same parameters were used and transmission ratio is:

$\frac{\omega_{\mathrm{o}}}{\omega_{\mathrm{i}}}=\frac{\Delta}{\mathrm{R}_{\mathrm{o}}-\Delta}$

Parameter $\Delta$ can be changed from its minimum $\Delta_{\text {min }}$ to its maximum $\Delta_{\max }$ value. In one prototype we have
$\mathrm{R}_{0}=65 \mathrm{~mm}, \Delta_{\max }=35 \mathrm{~mm}$ and $\Delta_{\min }=5 \mathrm{~mm}$ so the transmission ratio is:

For A mode from Eq. (1):

$\left.\frac{\omega_{\mathrm{o}}}{\omega_{\mathrm{i}}}\right|_{\min }=\left.0.071 \quad \frac{\omega_{\mathrm{o}}}{\omega_{\mathrm{i}}}\right|_{\max }=0.35$

For B mode from Eq. (2):

$\left.\frac{\omega_{\mathrm{o}}}{\omega_{\mathrm{i}}}\right|_{\min }=\left.0.083 \quad \frac{\omega_{\mathrm{o}}}{\omega_{\mathrm{i}}}\right|_{\max }=1.17$

The range of transmission ratio for two boundary values of $\Delta$ is: 
For A mode:

$\mathrm{T}=\frac{\left.\frac{\omega_{\mathrm{o}}}{\omega_{\mathrm{i}}}\right|_{\Delta \text { max }}}{\left.\frac{\omega_{\mathrm{o}}}{\omega_{\mathrm{i}}}\right|_{\Delta_{\text {min }}}}=\rightarrow \mathrm{T}_{\mathrm{A}}=\frac{\Delta_{\text {max }}}{\Delta_{\text {min }}} \cdot \frac{\mathrm{R}_{0}+\Delta_{\text {min }}}{\mathrm{R}_{0}+\Delta_{\text {max }}}=4.9$

For B mode:

$\mathrm{T}_{\mathrm{B}}=\frac{\Delta_{\max }}{\Delta_{\min }} \cdot \frac{\mathrm{R}_{0}-\Delta_{\min }}{\mathrm{R}_{0}-\Delta_{\max }}=14.1$

That indicates: $\mathrm{T}_{\mathrm{A}}<\mathrm{T}_{\mathrm{B}}$.

These results indicate that new CVT has wide transmission ratio that is larger than toroidal $[11,20]$ and belt $[22$, 23] CVTs those can provide about 2.5 speed ratio in maximum case.

By precisely adjusting the minimum value of $\Delta$, we can expand the transmission ratio to receive IVT functionality. For example, in the case of minimum free shaft radially displacement to be reduced to $\Delta_{\min }=1 \mathrm{~mm}$, the range of transmission ratio in $B$ mode will receive $T=74.6$. For $\mathrm{R}_{0}=65(\mathrm{~mm})$ curve of transmission ratio for both mode $A$ and $B$ are shown in Fig. 6.

\subsection{Transmission ratio exact solution}

In Fig. 4 parameter " $a$ " is free to vary from zero to $2 \pi$ radian as free shaft rotates. Follower's angle " $\beta$ " is a function of a as:

$\tan (\beta)=\frac{\Delta \sin (\alpha)}{R_{0}-\Delta \cos (\alpha)}$

If $-\pi / 2<a<\pi / 2$ this relation describes $B$ mode. If $\pi / 2<\alpha<3 \pi / 2$ this relation describes $A$ mode. Notice that in both modes $\beta$ takes all values, but because of the one-way clutch implementation, only some angular are important

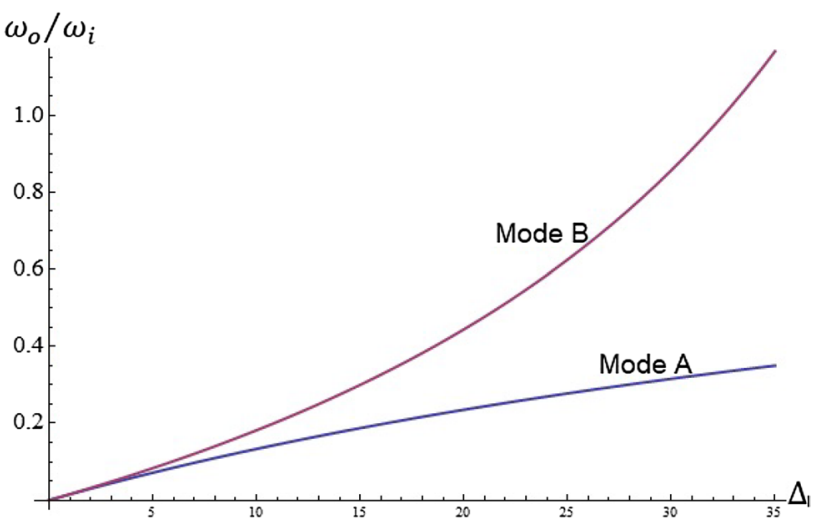

Fig. 6 Maximum transmission ratio for different $\Delta$ and $\mathrm{R}_{0}=65(\mathrm{~mm})$

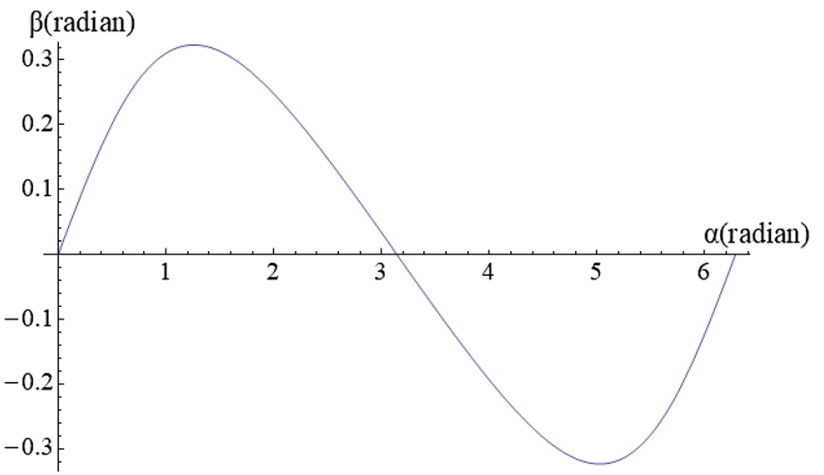

Fig. 7 Follower's angle for $\Delta=20(\mathrm{~mm})$ and $\mathrm{R}_{0}=65(\mathrm{~mm})$ as a function of rail angle

in every mode. For constant $\Delta=20(\mathrm{~mm})$ and $\mathrm{R}_{0}=65(\mathrm{~mm})$ curve of $\beta$ is shown in Fig. 7.

As we know $\omega_{i}=d a / d t$, that " $t$ " indicates time. And $\omega_{\mathrm{o}}=\mathrm{d} \beta / \mathrm{dt}$ is follower angular velocity. By taking time derivative from the $\beta$, we receive transmission ratio full description formula:

$\frac{\omega_{\mathrm{o}}}{\omega_{\mathrm{i}}}=\frac{\left(R_{0} \cos \alpha-\Delta\right) \Delta}{R_{0}^{2}+\Delta^{2}-2 R_{0} \Delta \cos \alpha}$

This relation gives us the followers angular velocity in different position of free shaft. For $-\pi / 2<a<\pi / 2$ this relation describes $B$ mode and for $\pi / 2<a<3 \pi / 2$ this relation describes $A$ mode. Follower's angular velocity $\omega_{0}$, for four different amounts of $\Delta$ and $R_{0}=65(\mathrm{~mm})$ and $\omega_{i}=1(\mathrm{rpm})$ is shown in Fig. 8.

Based on the Fig. 8, for one CVT prototype with four followers and $\Delta=10 \mathrm{~mm}$ in worst condition that each follower be engaged between angles $-\pi / 4<\alpha<\pi / 4$, the output rotational velocity will be in the form of Fig. 9 . Implementing spring clutch make the output smoother. Operation of CVT will be more reliable and stable by using more followers that reduces the engagement angle. Another way to have stable ratio is to force free-shaft to slides slowly on rails. This prevents sudden displacement of free shaft.

\section{Power losses in new CVT}

Main portion of power losses in new CVT is due to oscillatory motion of followers. As the follower get oscillate, it transfers the power in half of the oscillation path and then in the return half path, it acts as a loss component.

The power loss is related to the kinetic energy of oscillator: $1 / 2 m \omega^{2} x^{2}$, here $m$ is mass and $x$ is the oscillator 

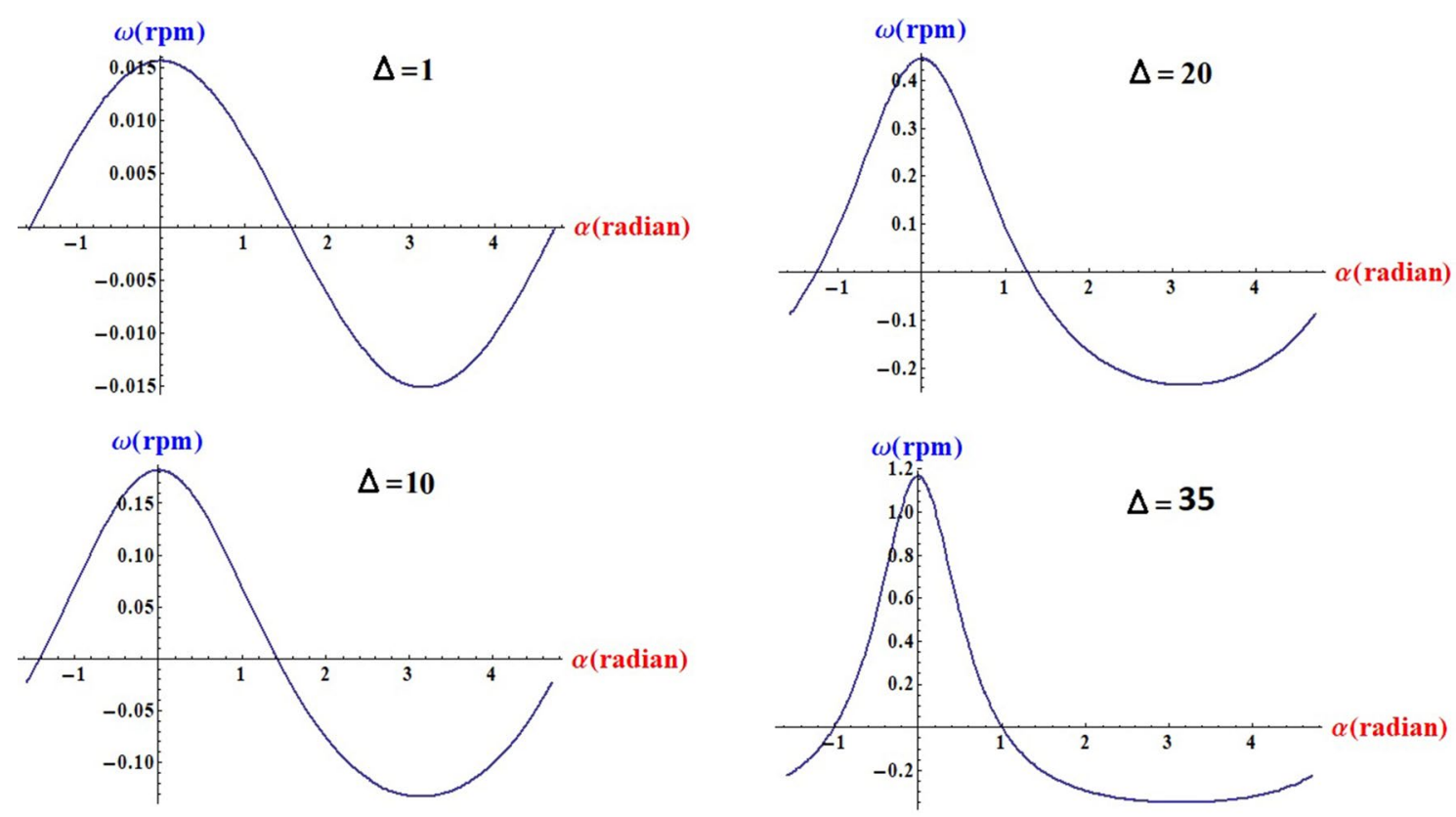

Fig. 8 Follower's angular velocity for different delta as a function of rail angle and $R_{0}=65(\mathrm{~mm})$ and $\omega_{i}=1(\mathrm{rpm})$

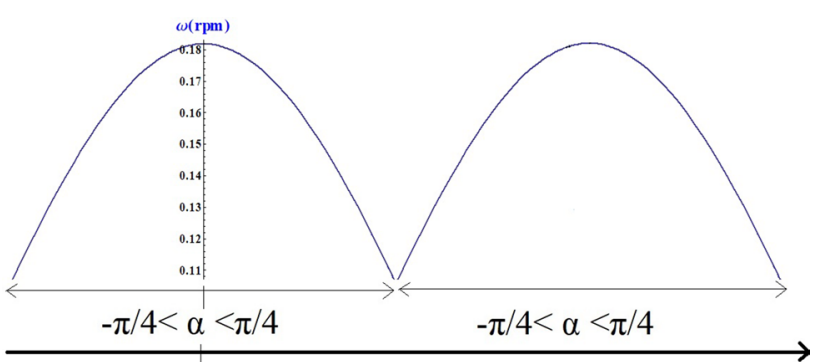

Fig. 9 Rotational velocity output of CVT with four followers that engaged between $-\pi / 4<\alpha<\pi / 4$, the followers sweep between these two ranges like pendulum

amplitude. One can lower the power loss by making followers from light materials.

In this context oscillator amplitude is shown by $\Delta$. Different transmission ratios are related to different $\Delta$, so the power loss of CVT is not constant for all transmission ratios. By the way there is a trade off between high transmission ratio and loss. But one proper design that make minimum $\Delta$ as small as possible, will receive the lowest power loss.

\section{Conclusion and suggestions}

Against cam-based CVTs, this new CVT mechanism could produce uniform output and it has no high stress limitation. Because in this new mechanism there is no cam and all followers are free to have any oscillation amplitudes.
Also, followers are not in high stress contact. So, this CVT change the gear ratio uniformly in wide range without any limitation on torque and speed. This CVT is self-adjustable, stable and reliable.

Authors' contributions This research and invention all done by Abbas Olyaei.

Availability of data and material Result are in agreement with simple handmade model.

\section{Compliance with ethical standards}

Conflict of interest The authors declare that they have no competing interests.

\section{References}

1. Xia Yu, Sun Dongye (2018) Characteristic analysis on a new hydro-mechanical continuously variable transmission system. Mech Mach Theory 126:457-467. https://doi.org/10.1016/j. mechmachtheory.2018.03.006

2. Li Q, Liao M, Wang S (2018) A zero-spin design methodology for transmission components generatrix in traction drive continuously variable transmissions. J Mech Des 140:033301. https:// doi.org/10.1115/1.4038646

3. Dutta-Roy T, Zhang N (2004) Effect of a half-toroidal continuously variable unit on the dynamics of a complete powertrain: a parametric free vibration analysis. Proc IMechE Part D: J Automob Eng 218(5):471-484. https://doi.org/10.1243/0954407047 74061138 
4. Ivanov K (2014) Automatic-box (CVT) without hydraulics. Am J Mech Appl 2(6-1):13-20. https://doi.org/10.11648 /j.ajma.s.2014020601.13

5. Hofman T, Steinbuch M, van Druten R, Serrarens AF (2008) Design of CVT-based hybrid passenger cars. IEEE Trans Veh Technol 58(2):572-587. https://doi.org/10.1109/TVT.2008.926217

6. Delkhosh M, Foumani S (2013) Multi-objective geometrical optimization of full toroidal CVT. Int J Automot Technol 14(5):707715. https://doi.org/10.1007/s12239-013-0077-0

7. Cretu OS, Glovnea RP (2005) Constant power continuously variable transmission (CP-CVT): operating principle and analysis. Trans ASME J Mech Des. https://doi.org/10.1115/1.1828457

8. Xinbo C, Peng H, Wei W, Yan L (2017) Design and analysis of a novel wheel type continuously variable transmission. Mech Mach Theory 107:13-26. https://doi.org/10.1016/j.mechmachth eory.2016.08.012

9. Carbone G, Novellis LD, Commissaris G, Steinbuch M (2010) An enhanced CMM model for the accurate prediction of steadystate performance of CVT chain drives. J Mech Des 132:021005. https://doi.org/10.1115/1.4000833

10. Zhang W, Zhang C, Guo W, Xiaobin X, Zhengxiong L (2017) Research on modeling and bending stress distribution of a new metal belt continuously variable transmission. Mech Mach Theory 116:220-233. https://doi.org/10.1016/j.mechmachth eory.2017.05.022

11. Carbone G, Mangialardi L, Mantriota G (2004) A comparison of the performances of full and half toroidal traction drives. Mech Mach Theory 39:921-942. https://doi.org/10.1016/j.mechm achtheory.2004.04.003

12. Yildiz A, Kopmaz O (2015) Dynamic analysis of a mechanical press equipped with a half-toroidal continuously variable transmission. Int J Mater Prod Technol 50(1):22-36

13. Patil KP, Jagadale KM, Patil BS, Mulik P, Cam Based IVT. IOSR J Mech Civ Eng (IOSR-JMCE), (ICETET-09), ISSN: 2278-1684, pp $13-20$

14. Jungyun K, Park FC, Park P, Shizuo M (2002) Design and analysis of a spherical continuously variable transmission. J Mech Des 124:21. https://doi.org/10.1115/1.1436487

15. Zhu C, Liu H, Tian J, Xiao Q, Du X (2010) Experimental investigation on the efficiency of the pulley-drive CVT. Int J Automot Technol 11(2):257-261. https://doi.org/10.1007/s1223 9-010-0032-2

16. Ryu W, Kim H (2007) Belt-pulley mechanical loss for a metal belt continuously variable transmission. Proc IMechE Part D: J Automob Eng. https://doi.org/10.1243/09544070JAUTO178

17. Junlong L, Dongye S, Ming Y, Xiaojun L, Baogang L (2018) Study on the transmission efficiency of electro-mechanical continuously variable transmission with adjustable clamping force. Mech Mach Theory 126:468-478. https://doi. org/10.1016/j.mechmachtheory.2018.04.012

18. Chen I-M, Huang Y-Y, Yang T-H, Liu T (2017) Limited-slip and torque-vectoring effect of a dual continuously variable transmission. Proc IMechE Part D: J Automob Eng. https://doi. org/10.1177/0954407016639465

19. Kim S, Moore C, Peshkin M, Colgate JE (2008) Causes of microslip in a continuously variable transmission. J Mech Des 130:011010. https://doi.org/10.1115/1.2803711

20. De Novellis L, Carbone G, Mangialardi L (2012) Traction and efficiency performance of the double roller full-toroidal variator: a comparison with half- and full-toroidal drives. J Mech Des 134:071005

21. Chen $X$, Hanga $P$, Wanga W, Li Y (2017) Design and analysis of a novel wheel type continuously variable transmission. Mech Mach Theory 107:13-26. https://doi.org/10.1016/j.mechmachth eory.2016.08.012

22. Yildiz A, Kopmaz O (2017) Control-oriented modelling with experimental verification and design of the appropriate gains of a PI speed ratio controller of chain CVTs. J Mech Eng 63(6):374382. https://doi.org/10.5545/sv-jme.2016.4184

23. Srivastava N, Haque I (2009) A review on belt and chain continuously variable transmissions (CVT): dynamics and control. J Mech Mach Theory 44:19-41. https://doi.org/10.1016/j.mechm achtheory.2008.06.007

24. Yildiz A, Piccininni A, Bottiglione F, Carbone G (2016) Modeling chain continuously variable transmission for direct implementation in transmission control. Mech Mach Theory 105:428-440. https://doi.org/10.1016/j.mechmachtheory.2016.07.015

25. Klovstad JW, Fortune JA (1997) Mechanical transmission continuously variable from forward to reverse. Patent 5603240

26. Patil KP, Gambhire VR (2014) New trend in infinitely variable transmission system based on cam. IGIRSET J. https://doi. org/10.15680/IJIRSET.2014.0308029

27. Lahr DF, Hong DW (2009) Operation and kinematic analysis of a CAM-based infinitely variable transmission. J Mech Des 131:081009. https://doi.org/10.1115/1.3179004

Publisher's Note Springer Nature remains neutral with regard to jurisdictional claims in published maps and institutional affiliations. 